\title{
Correction to: Clinical manifestations of Behçet's disease in a large cohort of Chinese patients: gender- and age-related differences
}

\author{
Chaoran $\mathrm{Li}^{1,2} \cdot \mathrm{Lu} \mathrm{Li}^{1,2} \cdot$ Xiuhua Wu $\mathrm{Wu}^{1,2,3} \cdot$ Jing Shi ${ }^{1,2} \cdot$ Jinjing Liu ${ }^{1,2} \cdot$ Jiaxin Zhou ${ }^{1,2} \cdot \mathrm{Li} \mathrm{Wang}^{1,2} \cdot$ Xinping Tian ${ }^{1,2}$. \\ Xiaofeng Zeng ${ }^{1,2} \cdot$ Wenjie Zheng ${ }^{1,2}$
}

Published online: 9 June 2020

(C) International League of Associations for Rheumatology (ILAR) 2020

\section{Correction to: Clinical Rheumatology https://doi.org/10.1007/s10067-020-05026-2}

The following Funding information was incomplete in the original publication; the authors have requested that this be noted. The complete information is as follows

Funding information This study was supported by grants from the National Key Research and Development Program: "Precise Medical Research" [2016YFC0906201], National Natural Science Foundation of China [81871299], the CAMS Innovation Fund for Medical Sciences [CIFMS 2016-I2M-1013, CIFMS2017-I2M-1-008 and CIFMS2019-I2M-2-008], and the Chinese National Key Technology R\&D Program, Ministry of Science and Technology [2017YFC0907601].

The remainder of the article remains unchanged.

Publisher's note Springer Nature remains neutral with regard to jurisdictional claims in published maps and institutional affiliations.

The online version of the original article can be found at https://doi.org/ $10.1007 / \mathrm{s} 10067-020-05026-2$

Wenjie Zheng

wenjzheng@gmail.com

Xiaofeng Zeng

xiaofeng.zeng@cstar.org.cn

1 Department of Rheumatology and Clinical Immunology, Peking Union Medical College Hospital, Chinese Academy of Medical Sciences \& Peking Union Medical College, Beijing 100730, China

2 National Clinical Research Center for Dermatologic and Immunologic Diseases (NCRC-DID), Key Laboratory of Rheumatology \& Clinical Immunology, Ministry of Education, Beijing 100730, China

3 Department of Rheumatology, General Hospital of Tianjin Medical University, Tianjin 300050, China 